TFT were normal. Bone marrow biopsy revealed mild aplasia, and no myelodysplastic syndrome or leukaemia. HIV, Hepatitis screen and viral serology were negative. Fanconi Anaemia test came back positive.

Conclusions Children presenting with macrocytosis should be carefully evaluated and referred to a haematologist, as it could be the first manifestation of a serious underlying bone marrow problem.

Macrocytosis in age range of six months to 12 years age is $\mathrm{MCV}>90$.

Causes of macrocytosis include disorders of folate/B12 metabolism, liver disease, congenital heart disease, Down's syndrome, hypothyroidism, drugs (anticonvulsants, zidovudine, hydroxyurea, immunosuppresants), myelodysplastic syndromes, bone marrow failure, and rare genetic syndromes (eg, Fanconi anaemia, thiamine-responsive megaloblastic anaemia syndrome).

\section{G408(P) CYTOKINE STORM ASSOCIATED MULTI-ORGAN FAILURE WITH POOR NEUROLOGICAL OUTCOME, DURING RITUXIMAB ADMINISTRATION IN A CHILD WITH RELAPSED ACUTE LYMPHOBLASTIC LEUKAEMIA AND EBV RELATED LYMPHOPROLIFERATIVE DISEASE}

${ }^{1} \mathrm{C}$ Kanaris, ${ }^{2} \mathrm{RF}$ Wynn, ${ }^{3} \mathrm{~A}$ Konstantinidis. ${ }^{1}$ Paediatric Intensive Care Unit, Royal Manchester Children's Hospital, Manchester, UK; ${ }^{2}$ Bone Marrow Transplant Unit, Royal Manchester Children's Hospital, Manchester, UK; ${ }^{3}$ Paediatric Haematology Department, Royal Manchester Children's Hospital, Manchester, UK

\subsection{6/archdischild-2015-308599.362}

We present the case of a 6 year old boy, with relapsed Acute Lymphoblastic Leukaemia and subsequent matched unrelated bone marrow transplant. His Epstein Barr Virus (EBV) titres were significant 6 months post-transplant with evidence of EBV related lymphoproliferative disease. Following local protocols, Rituximab was used for therapy and within minutes of starting the infusion the patient suffered pyrexia, hypotension and seizures with subsequent multi-organ failure, due to a cytokine storm. Organ damage included fulminant hepatic failure, renal failure meriting dialysis, cardiac failure requiring inotropic support and a significant acute brain injury. There was significant neurological impairment with radiological and electrophysiological evidence of global brain cell damage. As a result of irreversible neurological injury, the focus of treatment was switched from curative to palliative.

Cytokine storm is an immune mediated phenomenon, characterised by an overwhelming release of cytokines. This can produce a sepsis like response and may lead to multi-organ failure. Cytokines are normally produced by leucocytes as a response to an infective or inflammatory process and their main role is to induce leukopoeisis.

Rituximab is an anti-CD20 monoclonal antibody that has been useful in treating EBV related lymphoproliferative disease. Rituximab triggers the rapid release of cytokines that may lead to a cytokine storm with maximum levels within $2 \mathrm{~h}$ from the start of the infusion.

Cytokine storms are characterised by overproduction of immune mediators that in turn lead to cellular overactivation, increased endothelial permeability, polymorphonuclear neutrophil adhesion and migration. As there is lack of regulatory intervention, tissue congestion with activated leucocytes ensues which eventually causes parenchymal injury. Although we report multiorgan failure in this case, lungs and gut are the organs that are more commonly affected.
The incidence of cytokine storm in paediatric oncology patients is not known. It has been associated with haemophagocytic lymphohistiocytosis, graft-versus-host disease following haemopoetic stem cell transplant and the use of certain monoclonal antibodies. As in this case, results of the cytokine storm can be catastrophic, despite early recognition.

\section{G409(P) AN EVALUATION OF THE TRANSITION TO ADULT CARE FOR YOUNG PATIENTS WITH SICKLE CELL DISEASE}

${ }^{1} \mathrm{H}$ Sivaguru, ${ }^{2} \mathrm{~S}$ Mohun Kemp, ${ }^{2} \mathrm{R}$ Crowley, ${ }^{2} \mathrm{G}$ Hann, ${ }^{2} \mathrm{DA}$ Yardumian, ${ }^{2} \mathrm{M}$ RobertsHarewood, ${ }^{2} \mathrm{O}$ Wilkey. ${ }^{1}$ University College London, London, UK; ${ }^{2}$ North Middlesex University Hospital, London, UK

\subsection{6/archdischild-2015-308599.363}

Aims More than 95\% of children with sickle cell disease (SCD) survive into adulthood, making a successful transition from child to adult care an essential process. Poor management of this transition may result in reduced compliance, high non-attendance rates and adverse effects in later life. This study aimed to investigate the views of young patients in the process of transitioning with the long-term goal of improving previously poor engagement within the adolescent population.

Methods A questionnaire was given to all SCD patients between the ages of 13 and 21 who attended out-patient clinics over a four-month period.

Results 31 questionnaire responses were collected (response rate 94\%).

Overall satisfaction with the transition process varied with age. The mean score was 7.4/10 among 16-21 year olds, but only 4/10 among 13-15 year olds.

Praise was given for transition services such as a previously organised tour of the adult department, a peer discussion day and an information pack.

$48.4 \%$ were interested in speaking to peers about their experiences.

$41.9 \%$ reported receiving a transition information pack of which $81 \%$ found it useful.

Adolescents recounted that the topic of transitioning had been first broached at a wide range of ages (see Figure 1).

Conclusion There are discrepancies between the planned transition programme and reported patient experience. The tour of adult department and peer discussion day were praised, however not every adolescent recounted experiencing these services. Feedback from clinicians suggests that even though adolescents like the idea of these transition services they still fail to attend. A focus group has been initiated to improve adolescent

At what age did you hear about transistioning from children's to adult care?

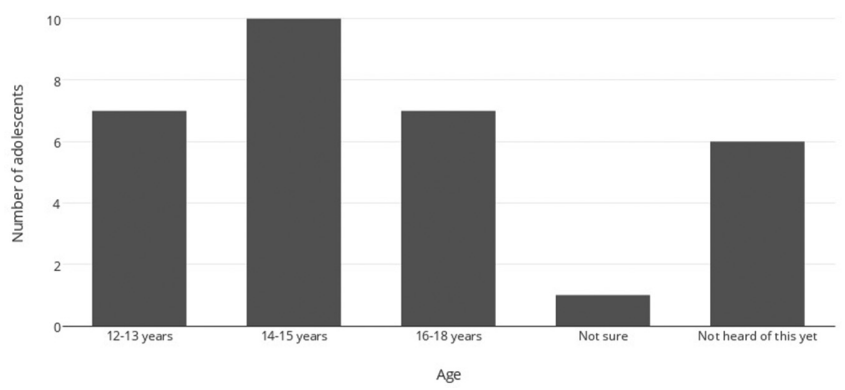

Abstract G409(P) Figure 1 